\title{
Lower Bounds for the Number of Bends in Three-Dimensional Orthogonal Graph Drawings
}

\author{
David R. Wood ${ }^{\star}$ \\ Basser Department of Computer Science \\ The University of Sydney \\ Sydney NSW 2006, Australia \\ davidw@cs.usyd.edu.au
}

\begin{abstract}
In this paper we present the first non-trivial lower bounds for the total number of bends in 3-D orthogonal drawings of maximum degree six graphs. In particular, we prove lower bounds for the number of bends in 3-D orthogonal drawings of complete simple graphs and multigraphs, which are tight in most cases. These result are used as the basis for the construction of infinite classes of $c$-connected simple graphs and multigraphs $(2 \leq c \leq 6)$ of maximum degree $\Delta(3 \leq \Delta \leq 6)$ with lower bounds on the total number of bends for all members of the class. We also present lower bounds for the number of bends in general position 3 -D orthogonal graph drawings. These results have significant ramifications for the '2-bends' problem, which is one of the most important open problems in the field.
\end{abstract}

\section{Introduction}

The 3-D orthogonal grid consists of grid-points in 3-space with integer coordinates, together with the axis-parallel grid-lines determined by these points. A 3-D orthogonal drawing of a graph places the vertices at grid-points and routes the edges along sequences of contiguous segments of grid-lines. Edges are allowed to contain bends and can only intersect at a common vertex. 3-D orthogonal drawings have been studied in 2345678121314 . For brevity we say a 3-D orthogonal graph drawing is a drawing. A drawing with no more than $b$ bends per edge is called a b-bend drawing. The graph-theoretic terms 'vertex' and 'edge' also refer to their representation in a drawing. The ports at a vertex $v$ are the six directions, denoted by $\left\{X^{+}, X^{-}, Y^{+}, Y^{-}, Z^{+}, Z^{-}\right\}$, which the edges incident with $v$ can use. For each dimension $I \in\{X, Y, Z\}$, the $I^{+}$(respectively, $I^{-}$) port at a vertex $v$ is said to be extremal if $v$ has maximum (minimum) $I$-coordinate taken over all vertices. Clearly, orthogonal drawings can only exist for graphs with maximum degree six.

Drawings with many bends appear cluttered and are difficult to visualise. Therefore minimising the number of bends, along with minimising the bounding box volume, have been the most commonly proposed aesthetic criteria for

\footnotetext{
* Supported by the Australian Research Council Large Grant A49906214.

J. Marks (Ed.): GD 2000, LNCS 1984, pp. 259-272, 2001.

(c) Springer-Verlag Berlin Heidelberg 2001
} 
measuring the quality of a drawing. Using straightforward extensions of the corresponding 2-D NP-hardness results, optimising each of these criteria is NP-hard [4]. Kolmogorov and Barzdin [6] established a lower bound of $\Omega\left(n^{3 / 2}\right)$ for the bounding box volume of a drawings of $n$-vertex graphs. In this paper we establish the first non-trivial lower bounds for the number of bends in 3-D orthogonal drawings. Lower bounds for the number of bends in 2-D orthogonal graph drawings have been established by Tamassia et al. [9] and Biedl [1].

Lower bounds for the maximum number of bends per edge: Obviously every drawing of $K_{3}$ has at least one bend. It follows from results in multi-dimensional orthogonal graph drawing by Wood [11] that every drawing of $K_{5}$ has an edge with at least two bends. It is well known that every drawing of $6 K_{2}$ has an edge with at least three bends, and easily seen that $2 K_{2}$ and $3 K_{2}$ have at least one edge with at least one and two bends, respectively. Here $k K_{2}$ is the 2-vertex multigraph with $k$ edges.

A natural candidate for the existence of a simple graph with a 3-bend edge in every drawing is $K_{7}$, as originally conjectured by Eades et al. [5]. A counterexample to this conjecture, namely a drawing of $K_{7}$ with at most two bends per edge, was first exhibited by Wood 11. A more symmetric drawing of $K_{7}$ with at most two bends per edge is shown in Fig. П(a). This drawing has the interesting feature of rotational symmetry about the line $X=Y=Z$. One may consider the other 6-regular complete multi-partite graphs $K_{6,6}, K_{3,3,3}$ and $K_{2,2,2,2}$ to be potential examples of simple graphs with a 3 -bend edge in every drawing. 2-bend drawings of these graphs are presented in [14.
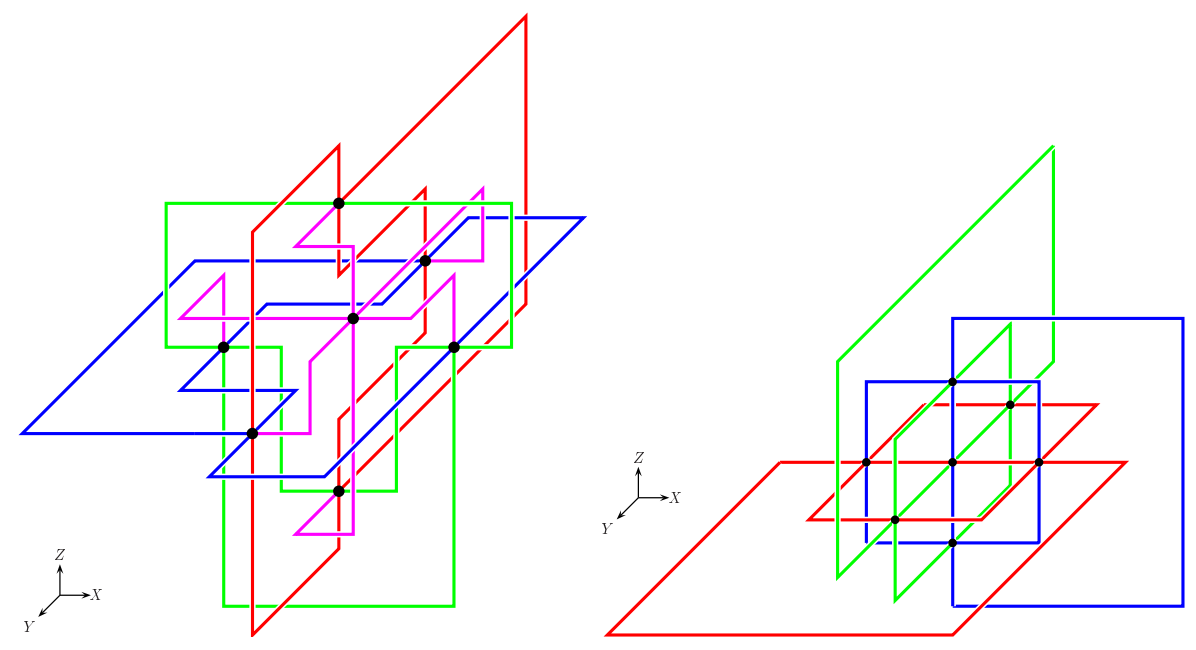

Fig. 1. (a) 2-bend drawing of $K_{7}$ (b) 4-bend drawing of $K_{7}$ with 24 bends.

Lower bounds for the total number of bends: In this paper we prove that drawings of the complete graphs $K_{4}, K_{5}, K_{6}$ and $K_{7}$ have at least $3,7,12$ and 20 bends, respectively. For each of these graphs except $K_{7}$ there are well- 
known drawings with the corresponding number of bends. Figure 1(b) shows a drawing of $K_{7}$ with a total of 24 bends (compared with the total of 42 bends for the 2-bend drawing). We conjecture that there is no drawing of $K_{7}$ with fewer than 24 bends.

We use these lower bounds for the number of bends in complete graphs as the basis for the construction of infinite families of $c$-connected graphs of maximum degree $\Delta$ with lower bounds on the number of bends for each member of the class. Table 1 summarises these lower bounds.

Table 1. Lower bounds for the number of bends in drawings of $m$-edge $c$-connected graphs with maximum degree $\Delta$.

\begin{tabular}{c|cccc|cccc}
\hline \hline \multirow{2}{*}{ Connectivity $c$} & \multicolumn{4}{|c|}{ Simple Graphs } & \multicolumn{4}{c}{ Multigraphs } \\
\cline { 2 - 9 } & $\Delta=6$ & $\Delta=5$ & $\Delta=4$ & $\Delta=3$ & $\Delta=6$ & $\Delta=5$ & $\Delta=4$ & $\Delta=3$ \\
\hline \hline 0 & $\frac{20}{21} m$ & $\frac{4}{5} m$ & $\frac{7}{10} m$ & $\frac{1}{2} m$ & $2 m$ & $\frac{8}{5} m$ & $\frac{3}{2} m$ & $\frac{4}{3} m$ \\
2 & $\frac{3}{4} m$ & $\frac{7}{11} m$ & $\frac{3}{7} m$ & $\frac{1}{4} m$ & $\frac{4}{3} m$ & $\frac{6}{5} m$ & $m$ & $\frac{2}{3} m$ \\
3 & $\frac{8}{11} m$ & $\frac{14}{23} m$ & $\frac{2}{5} m$ & $\frac{2}{9} m$ & $m$ & $\frac{4}{5} m$ & $\frac{1}{2} m$ & - \\
4 & $\frac{12}{17} m$ & $\frac{7}{12} m$ & $\frac{3}{8} m$ & - & $\frac{2}{3} m$ & $\frac{2}{5} m$ & - & - \\
5 & $\frac{24}{35} m$ & $\frac{14}{25} m$ & - & - & $\frac{1}{3} m$ & - & - & - \\
6 & $\frac{2}{3} m$ & - & - & - & - & - & - & - \\
\hline \hline
\end{tabular}

Upper bounds: A number of algorithms have been proposed for 3-D orthogonal graph drawing [2]3,5,6,8,12,14] which explore the apparent tradeoff between the maximum number of bends per edge and the bounding box volume (see [14] for an overview). We now summarise the known upper bounds on the number of bends in the drawings produced by these algorithms. The 3-BENDS algorithm of Eades et al. [5] and the InCREMENTAL algorithm of Papakostas and Tollis [7] both produce 3-bend drawing 1$]$ of multigraph 2 with maximum degree six. As discussed above there exists simple graphs with at least one edge having at least two bends in every drawing. The following problem is therefore of considerable interest:

2-Bends Problem: Does every simple graph with maximum degree six admit a 2-bend drawing? [5]

\footnotetext{
${ }^{1}$ The 3 -BENDS algorithm [5] produces drawings with $27 n^{3}$ volume. By deleting gridplanes not containing a vertex or a bend the volume is reduced to $8 n^{3}$. The INCREMENTAL algorithm [7] produces drawings with $4.63 n^{3}$ volume. A modification of the 3 -BENDS algorithm by Wood [14] produces drawings with $n^{3}+o\left(n^{3}\right)$ volume.

2 The 3-Bends algorithm [5] explicitly works for multigraphs. The INCREMENTAL algorithm, as stated in [7, only works for simple graphs; with a suitable modification it also works for multigraphs [A. Papakostas, private communication, 1998].
} 
The Diagonal Layout and Movement algorithm of Wood [12] solves the 2-bends problem in the affirmative for simple graphs with maximum degree five. For maximum degree six simple graphs, the same algorithm uses a total of at most $7 \mathrm{~m} / 3$ bends, which is the best known upper bound for the total number of bends in 3-D orthogonal drawings.

In this paper we provide a negative result related to the 2-bends problem. A 3 -D orthogonal graph drawing is said to be in general position if no two vertices lie in a common grid-plane. The general position model is used in the 3-BENDS and Diagonal Layout and Movement algorithms. In this paper we show that the general position model, and the natural variation of this model where pairs of vertices share a common plane, cannot be used to solve the 2-bends problem, at least for 2-connected graphs.

The remainder of this paper is organised as follows. In Sect. 2 we establish a number of introductory results concerning 0-bend drawings of cycles. These results are used to prove our lower bounds on the total number of bends in drawings of complete graphs, established in Sect. 3. In Sect.4 we use these lower bounds as the basis for lower bounds on the number of bends in infinite families of graphs of varying connectivity and maximum degree. In Sect. 5 we present lower bounds for the number of bends in general position drawings and their implications for the 2-bends problem.

Throughout this paper we consider $n$-vertex $m$-edge loop-free graphs with maximum degree six. By $C_{m}$ we denote the $m$-edge cycle. A chord of a cycle $C$ is an edge not in $C$ whose end-vertices are both in $C$. We say two cycles are chord-disjoint if they do not have a chord in common. In this paper most proofs are outlined, and the proofs of the results in Table1 1 concerning multigraphs are omitted; see [13] for details of all the proofs.

\section{Drawings of Cycles}

In this section we characterise the 0-bend drawings of the cycles $C_{k}(k \leq 7)$. We then show that if a drawing of a complete graph contains such a 0-bend drawing of a cycle then there must be many edges with at least three bends in the drawing of the complete graph. These results are used in Sect. 3 in our lower bounds for the total number of bends in drawings of complete graphs.

A straight-line path in a 0-bend drawing of a cycle is called a side. A side parallel to the $I$-axis for some $I \in\{X, Y, Z\}$ is called an $I$-side, and $I$ is called the dimension of the side. Clearly the dimension of adjacent sides is different, thus in a 2-dimensional drawing the dimension of the sides alternate around the cycle. Hence, there is no 2-dimensional 0-bend drawing of a cycle with an odd number of sides. If there is an $I$-side in a drawing of a cycle for some $I \in\{X, Y, Z\}$ then clearly there is at least two $I$-sides. Therefore a drawing of a cycle with $X$-, $Y$ - and $Z$-sides, which we call truly 3-dimensional, must have at least six sides. Hence there is no truly 3 -dimensional 3-, 4- or 5-sided 0-bend drawing of a cycle. There is also no two-dimensional 3- or 5-sided 0-bend drawing of a cycle. We therefore have the following observation. 
Observation 1 (a) There is no 3- or 5-sided 0-bend drawing of a cycle, (b) there is no 0-bend drawing of $C_{3}$, and

(c) all 0-bend drawings of $C_{4}$ and $C_{5}$ have four sides.

The next result forms an important component of the lower bounds to follow.

Lemma 1. If a drawing of a complete graph contains a 0-bend 4-cycle (respectively, 5-cycle) then there are at least two (four) chords of the cycle each with at least three bends.

Proof. By Obs. 1(c) all 0-bend drawings of $C_{4}$ and of $C_{5}$ have four sides. As illustrated in Fig. 2(a), the chord connecting each pair of diagonally opposite vertices in a 4-sided drawing of a cycle has at least three bends. Hence, if a drawing of a complete graph contains a 0 -bend $C_{4}$, then the two chords each have at least three bends. Also, in the case of $C_{5}$, the two edges from the vertex not at the intersection of two sides to the diagonally opposite vertices both have at least three bends, as in Fig. 2(b). Hence, if a drawing of a complete graph contains a 0 -bend $C_{5}$, then the four chords each have at least three bends.

(a)

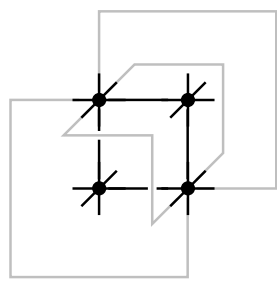

(b)

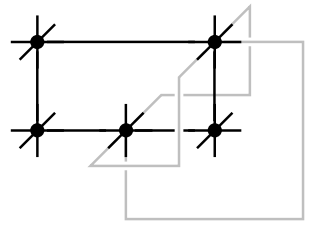

(c)

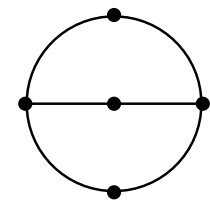

Fig. 2. 3-bend edges 'across' (a) $C_{4}$ and (b) $C_{5}$. (c) The graph $H_{1}$

Consider the graph shown in Fig. 2(c), which we call $H_{1}$.

Observation $2 H_{1}$ does not have a 0-bend drawing.

Proof. $H_{1}$ contains $C_{4}$. As in Lemma 1, an edge between the non-adjacent vertices of a 4 -sided cycle needs at least three bends. Hence the 2-path in $H_{1}$ between the non-adjacent vertices of the 4-cycle has at least one bend. Therefore $H_{1}$ does not have a 0-bend drawing.

We now consider 6-sided 0-bend drawings of a cycle.

Lemma 2. If a drawing of a complete graph contains a 0-bend 6-cycle then there are at least four chords of the cycle each with at least three bends.

Proof. We can assume without loss of generality that there is a 0-bend drawing of $C_{6}$ contained in a drawing of $K_{6}$. By Obs. 1(a), all 0-bend drawings of $C_{6}$ have four or six sides. In a 4 -sided 0-bend drawing of $C_{6}$ the two vertices not at the intersection of adjacent sides can be (a) on the same side, (b) on adjacent sides, or (c) on opposite sides. In each case there are at least six chords from a 
vertex at the intersection of two sides to a vertex on neither of these sides, each with at least three bends.

Case analysis shows that the only 6-sided 0-bend drawings of $C_{6}$ (up to symmetry) are those in Fig. 3. For each such drawing, the chords of $C_{6}$ shown in Fig. 3 each require at least three bends. In the case of the drawing in Fig. 3. (c) there are at least six chords each requiring at least three bends. For the drawing in Fig. 3(a) (respectively, Fig. 3(b)) it can be shown, by considering certain sets of chords for which all edge routes with at most two bends pass through a single grid point, that a further two (four) chords have at least three bends. Hence there are at least six chords of the cycle each with at least three bends.

(a)

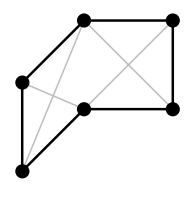

(b)

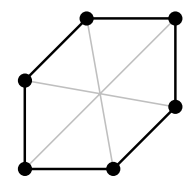

(c)

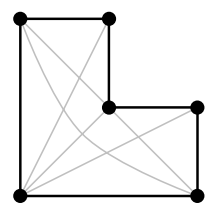

Fig. 3. Edges with at least 3 bends in a drawing of $K_{6}$ containing a 6 -sided 0-bend $C_{6}$.

We now consider 7-sided 0-bend drawings of a cycle.

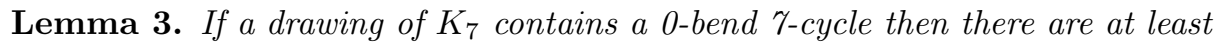
four chords of the cycle each with at least three bends.

Proof. By Obs. 1(a), a 0-bend drawing of $C_{7}$ has four, six or seven sides. In a 4-sided 0-bend drawing of $C_{7}$ the three vertices not at the intersection of two adjacent sides can be (a) all on the same side, (b) two on one side and one on an adjacent side, (c) two on one side and one on the opposite side, or (d) all on different sides. In each case there are at least eight chords from a vertex at the intersection of two sides to a vertex on neither of these sides, each with at least three bends. The 6-sided 0-bend drawings of $C_{7}$ can be obtained from the 6sided 0-bend drawings of $C_{6}$ by placing one new vertex at each of the essentially different edges of each drawing. By Lemma 2 at least six of the chords of $C_{6}$, and therefore of $C_{7}$, have at least three bends. Case analysis shows that the only 7-sided 0-bend drawings of $C_{7}$ are those shown in Fig. 4 For each drawing there are at least four chords which need at least three bends.

(a)

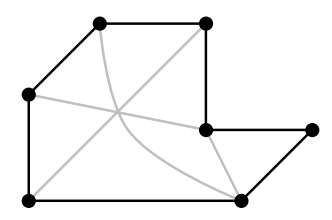

(b)

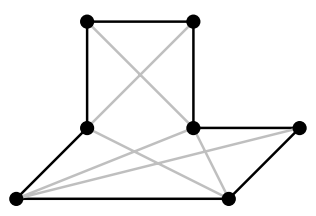

(c)

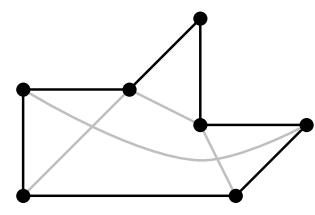

Fig. 4. Edges with at least three bends in a 7-sided 0-bend drawing of $C_{7}$. 


\section{Drawings of Complete Graphs}

In this section we establish lower bounds for the total number of bends in 3-D orthogonal drawings of $K_{4}, K_{5}, K_{6}$ and $K_{7}$. We omit our proofs that the wellknown drawings of $K_{4}, K_{5}$ and $K_{6}$ shown in Fig. 5 are bend-minimum. They are similar to the proof of the lower bound for $K_{7}$ which follows.

(a)

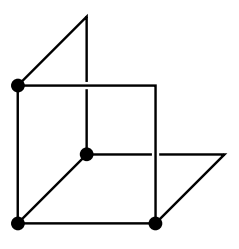

(b)

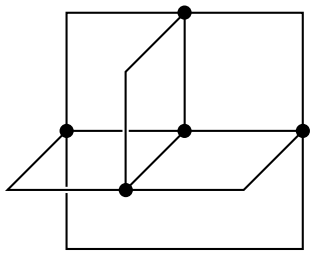

(c)

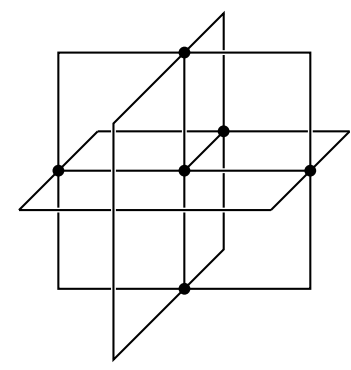

Fig. 5. (a) $K_{4}$ with 3 bends, (b) $K_{5}$ with 7 bends and (c) $K_{6}$ with 12 bends.

Theorem 1. Every drawing of $K_{4}$ has at least three bends. Every drawing of $K_{5}$ has at least seven bends. Every drawing of $K_{6}$ has at least twelve bends.

Figure 1(b) shows a drawing of $K_{7}$ with a total of 24 bends.

Theorem 2. Every drawing of $K_{7}$ has at least 20 bends.

Proof. Suppose to the contrary, that there is a drawing of $K_{7}$ with at most 19 bends. The subgraph of $K_{7}$ consisting of the 0-bend edges is called the 0-bend subgraph. Let $k_{i}(i \geq 0)$ be the number of $i$-bend edges. Hence

$$
\sum_{i \geq 0} k_{i}=21 \text {, and } \sum_{i \geq 1} i k_{i} \leq 19 .
$$

Case analysis shows that every subgraph of $K_{7}$ with at least ten edges contains $C_{3}$ or $H_{1}$. By Obs. 1 b) and Obs. 2 the graphs $C_{3}$ and $H_{1}$ do not have 0 -bend drawings. Hence $k_{0} \leq 9$. Suppose $k_{0}=8$ or $k_{0}=9$. By (1)

$$
\begin{aligned}
& 12 \leq \sum_{i \geq 1} k_{i} \leq 19-\sum_{i \geq 1}(i-1) k_{i} \\
& \sum_{i \geq 2}(i-1) k_{i} \leq 7 .
\end{aligned}
$$

Case analysis shows that every subgraph of $K_{7}$ with at least eight edges contains a cycle $C_{k}(k \neq 4)$, two chord-disjoint cycles, or an $H_{1}$ subgraph. Therefore the 0-bend subgraph contains a cycle $C_{k}(k \geq 5)$ or two chord-disjoint subgraphs (since $C_{3}$ and $H_{1}$ do not have 0-bend drawings by Obs. 1(b) and Obs. 2, respectively). If the 0-bend subgraph contains a cycle $C_{k}(k \geq 5)$ then by Lemma 1, Lemma 2 and Lemma 3 there are at least four chords of the 
cycle each with at least three bends. On the other hand if the 0-bend subgraph contains two chord-disjoint cycles then these cycles have length at least four; thus by Lemma 1, Lemma 2 and Lemma 3, two chords from each of these cycles each have at least three bends. In either case the drawing of $K_{7}$ has at least four edges each with at least three bends; that is,

$$
\begin{aligned}
4 & \leq \sum_{i \geq 3} k_{i} \\
k_{2}+8 & \leq k_{2}+\sum_{i \geq 3} 2 k_{i} \leq \sum_{i \geq 2}(i-1) k_{i} \\
k_{2}+8 & \leq 7
\end{aligned}
$$

Hence $k_{2} \leq-1$, which is a contradiction. Therefore $k_{0} \leq 7$. By (11) with $k_{0} \leq 7$

$$
\begin{aligned}
& 14 \leq \sum_{i \geq 1} k_{i} \leq 19-\sum_{i \geq 1}(i-1) k_{i} \\
& \sum_{i \geq 2}(i-1) k_{i} \leq 5 \\
& k_{2}+2 \sum_{i \geq 3} k_{i} \leq 5
\end{aligned}
$$

Let $A$ be the set of edges of $K_{7}$ routed using an extremal port at exactly one end-vertex. Let $B$ be the set of edges routed using extremal ports in the same direction at its end-vertices. Let $C$ be the set of edges routed using extremal ports in differing directions at its end-vertices. $K_{7}$ is 6 -regular, thus all ports are used. Since there is at least one extremal port in each direction, we have $|A|+|B|+2|C| \geq 6$. It is easily seen that an edge in $A$ or $B$ has at least two bends, and that an edge in $C$ has at least three bends. Hence

$$
k_{2}+2 \sum_{i \geq 3} k_{i} \geq 6
$$

This contradicts (3). The result follows.

\section{Constructing Large Graphs}

In this section we use the lower bounds for the total number of bends in drawings of the complete graphs established in Sect. 3 as building blocks to construct infinite families of graph with lower bounds for the number of bends.

Given graphs $G$ and $H$ with $|V(G)| \geq \Delta(H)$, we define $H\langle G\rangle$ to be the graph obtained by replacing each vertex of $H$ by a copy of $G$, and connecting the vertices adjacent to a vertex $v \in V(H)$ to different vertices in the copy of $G$ corresponding to $v$. In most cases, $H$ is regular and $G$ is a complete graph, thus $H\langle G\rangle$ is well-defined. In other cases we shall specify the mapping between edges incident to $v$ and the vertices in the copy of $G$ corresponding to $v$. We 
also employ the cartesian product $G \times H$ of graphs $G$ and $H$ to construct larger graphs. $G \times H$ has vertex set $V(H) \times V(G)$ with $\left(v_{1}, w_{1}\right)$ and $\left(v_{2}, w_{2}\right)$ adjacent in $G \times H$ if either $v_{1}=v_{2}$ and $w_{1} w_{2} \in E(H)$, or $w_{1}=w_{2}$ and $v_{1} v_{2} \in E(G)$.

By taking disjoint copies of $K_{7}, K_{6}, K_{5}$ and $K_{4}$ the next result follows immediately from Theorem 1 and Theorem 2

Theorem 3. There exists infinite families of simple (disconnected) m-edge graphs with maximum degree six (respectively, five, four and three) with at least $\frac{20}{21} m\left(\frac{4}{5} m, \frac{7}{10} m\right.$ and $\left.\frac{1}{2} m\right)$ bends in every drawing.

To obtain our lower bounds for 2-, 3- and 4-connected graphs consider the graphs $C_{r}\left\langle K_{p}\right\rangle(p \geq 3),\left(C_{r} \times K_{2}\right)\left\langle K_{p}\right\rangle(p \geq 3)$ and $\left(C_{r} \times C_{3}\right)\left\langle K_{p}\right\rangle(p \geq 4)$ for some $r \geq 3$, as illustrated in Fig. 6]

(a)

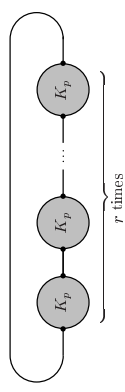

(b)

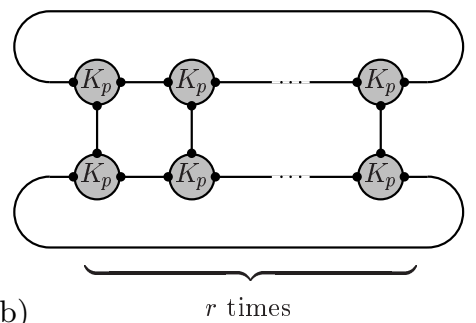

(b) 3-connected $\left(C_{r} \times K_{2}\right)\left\langle K_{p}\right\rangle$,

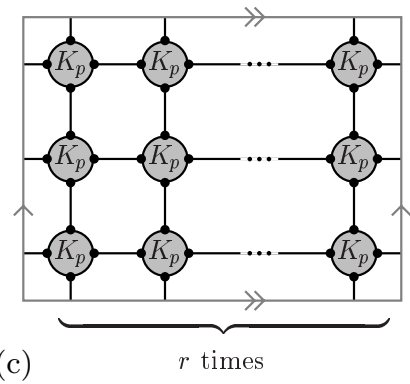

(c) 4-connected Fig. 6.
$\left(C_{r} \times C_{3}\right)\left\langle K_{p}\right\rangle$.

Theorem 4. There exists infinite families of simple 2-connected m-edge graphs with maximum degree six (respectively, five, four and three) with at least $\frac{3}{4} m$ $\left(\frac{7}{11} m, \frac{3}{7} m\right.$ and $\left.\frac{1}{4} m\right)$ bends in every drawing.

Proof. The graphs $C_{r}\left\langle K_{6}\right\rangle$ with $r \geq 2$ have maximum degree six and $m=16 r$ edges. By Theorem $1 K_{6}$ has at least 12 bends in every drawing, thus $C_{r}\left\langle K_{6}\right\rangle$ has at least $12 r=\frac{3}{4} m$ bends in every drawing. The graphs $C_{r}\left\langle K_{5}\right\rangle$ with $r \geq 2$ have maximum degree five and $m=11 r$ edges. By Theorem $1 K_{5}$ has at least 7 bends in every drawing, thus $C_{r}\left\langle K_{5}\right\rangle$ has at least $7 r=\frac{7}{11} m$ bends in every drawing. The graphs $C_{r}\left\langle K_{4}\right\rangle$ with $r \geq 2$ have maximum degree four and $m=7 r$ edges. By Theorem $1 K_{4}$ has at least 3 bends in every drawing, thus $C_{r}\left\langle K_{4}\right\rangle$ has at least $3 r=\frac{3}{7} m$ bends in every drawing. The graphs $C_{r}\left\langle K_{3}\right\rangle$ with $r \geq 2$ have maximum degree three and $m=4 r$ edges. By Obs. 1(b), $K_{3}$ has at least 1 bend in every drawing, thus $C_{r}\left\langle K_{3}\right\rangle$ has at least $r=\frac{1}{4} m$ bends in every drawing. Clearly $C_{r}\left\langle K_{p}\right\rangle$ is 2-connected.

The proofs of the following results for 3- and 4-connected graphs are similar to the proof of Theorem 4 
Theorem 5. There exists infinite families of simple 3-connected m-edge graphs with maximum degree six (respectively, five, four and three) with at least $\frac{8}{11} m$ $\left(\frac{14}{23} m, \frac{2}{5} m\right.$ and $\left.\frac{2}{9} m\right)$ bends in every drawing.

Theorem 6. There exists an infinite family of simple 4-connected $m$-edge graphs with maximum degree six (respectively, five and four) with at least $\frac{12}{17} \mathrm{~m}$ $\left(\frac{7}{12} m\right.$ and $\left.\frac{3}{8} m\right)$ bends in every drawing.

To obtain our lower bounds for 5 - and 6-connected graphs consider the graphs $\left(C_{r} \times C_{3} \times K_{2}\right)\left\langle K_{p}\right\rangle(p \geq 5)$ and $\left(C_{r} \times C_{3} \times C_{3}\right)\left\langle K_{6}\right\rangle$ for some $r \geq 3$, as illustrated in Fig. 7 Again the proofs are very similar to that of Theorem 4 .

(a)

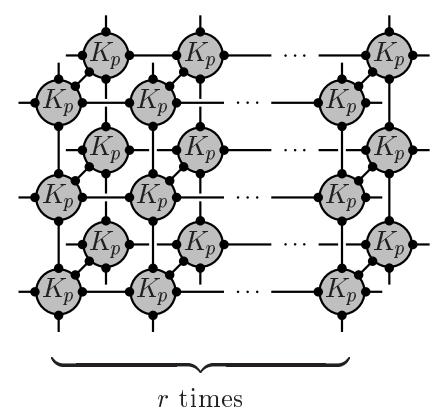

(b)

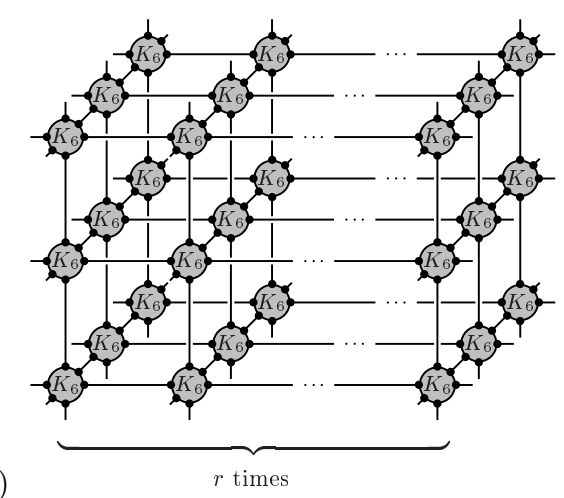

Fig. 7. (a) 5-connected $\left(C_{r} \times C_{3} \times K_{2}\right)\left\langle K_{p}\right\rangle$, (b) 6-connected $\left(C_{r} \times C_{3} \times C_{3}\right)\left\langle K_{6}\right\rangle$.

Theorem 7. There exists infinite families of simple 5-connected $m$-edge graphs with maximum degree six (respectively, five) with at least $\frac{24}{35} m\left(\frac{14}{25} m\right)$ bends in every drawing.

Theorem 8. There exists an infinite family of simple 6-connected m-edge graphs with maximum degree six with at least $\frac{2}{3} m$ bends in every drawing.

\section{General Position Drawings and the 2-Bends Problem}

Recall that a 3-D orthogonal graph drawing is in general position if no two vertices lie in a common grid-plane. In this section we establish lower bounds for the number of bends in general position drawings.

Lemma 4. If the graph $G$ has at least $k$ bends in every general position drawing then for any edge e of $G$ the graph $G \backslash e$ has at least $k-4$ bends in every general position drawing.

Proof. If $G \backslash e$ has a drawing with $b$ bends then, the edge $e$ can be inserted into the drawing with at most four bends and the edges rerouted so that there are no edge crossings [12,14]. Thus, there is a general position drawing of $G$ with $b+4$ bends. Every general position drawing of $G$ has at least $k$ bends, hence $b+4 \geq k$ and $b \geq k-4$. 
Clearly every edge in a general position drawing has at least two bends. The following lower bounds for general position drawings are based on the observation that an edge routed using an extremal port in a general position drawing has at least three bends. For 6 -regular $m$-edge graphs all ports must be used, thus such a graph has at least $2 m+6$ bends in every general position drawing. Hence the graphs consisting of disjoint copies of $K_{7}$ provide the following lower bound.

Lemma 5. There exists an infinite family of $n$-vertex $m$-edge simple graphs, each with at least $2 m+6 n / 7$ bends in every general position drawing.

Note that for 6 -regular graphs the above lower bound is within $m / 21$ of the upper bound of $7 \mathrm{~m} / 3$ for the total number of bends in general position drawings established by the Diagonal Layout and Movement algorithm [12].

To establish our lower bound for general position drawings of 2-connected graphs consider the graph $C_{r}\left\langle K_{7} \backslash e\right\rangle$ for $r \geq 2$, where the non-adjacent vertices of each $K_{7} \backslash e$ are incident to the edges of $C_{r}$, as illustrated in Fig. 8(a).

(a)

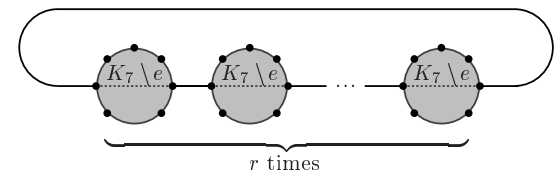

(b)

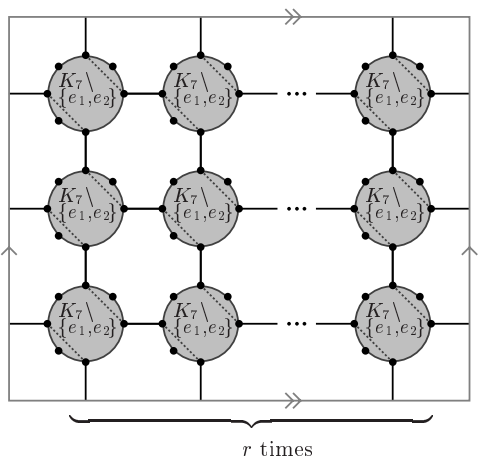

Fig. 8. (a) 2-connected $C_{r}\left\langle K_{7} \backslash e\right\rangle$, (b) 4-connected $\left(C_{r} \times C_{3}\right)\left\langle K_{7} \backslash\left\{e_{1}, e_{2}\right\}\right\rangle$.

Lemma 6. There is an infinite family of n-vertex $m$-edge simple 2-connected graphs, each with at least $2 m+4 n / 7$ bends in every general position drawing.

Proof. Clearly $C_{r}\left\langle K_{7} \backslash e\right\rangle$ is 2-connected. $K_{7}$ has at least $2\left|E\left(K_{7}\right)\right|+6$ bends in every general position drawing. Thus by Lemma 4, a general position drawing of $K_{7} \backslash e$ has at least $2\left|E\left(K_{7}\right)\right|+6-4=2\left|E\left(K_{7} \backslash e\right)\right|+4$ bends. The edges of $C_{r}$ each have at least two bends, thus $C_{r}\left\langle K_{7} \backslash e\right\rangle$ has at least $2 m+4 n / 7$ bends in every general position drawing.

To establish our lower bound for general position drawings of 4-connected graphs consider the graph $\left(C_{r} \times C_{3}\right)\left\langle K_{7} \backslash\left\{e_{1}, e_{2}\right\}\right\rangle$ for $r \geq 2$, where for each copy of $K_{7} \backslash\left\{e_{1}, e_{2}\right\}, e_{1}$ and $e_{2}$ are edges of $K_{7}$ with no common end-vertices, and these end-vertices are incident to different edges in $C_{r} \times C_{3}$, as illustrated in Fig. 8 (b).

The proof of the next result is very similar to that of Lemma 6 ,

Lemma 7. There is an infinite family of n-vertex $m$-edge simple 4-connected graphs, each with at least $2 m+2 n / 7$ bends in every general position drawing. 
We now look at the ramifications of the above general position lower bounds for the 2-bends problem. Edges with at most two bends can be classified as 0bend, 1-bend, 2-bend planar or 2-bend non-planar. As illustrated in Fig. 9 a given 2-bend drawing can be transformed into a general position drawing whose number of bends depends on the number of 0-bend and 2-bend planar edge routes in the 2-bend drawing. We omit the proof.

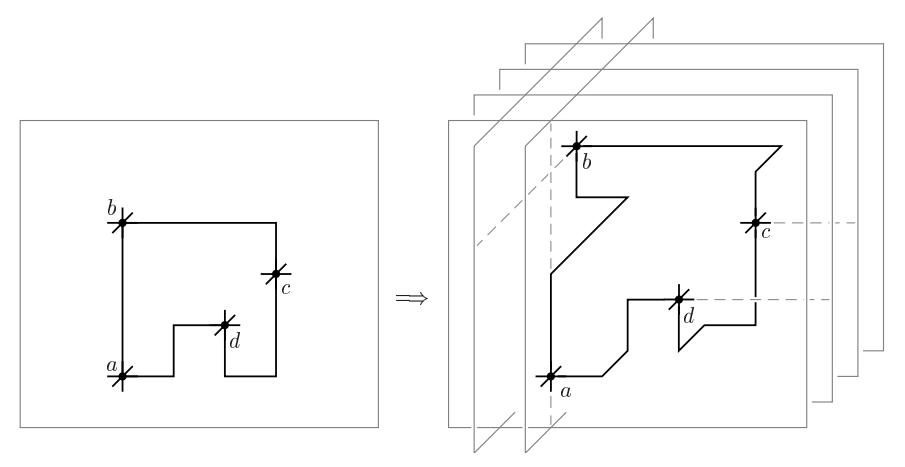

Fig. 9. Removing a plane containing many vertices.

Lemma 8. If in a 2-bend drawing of an m-edge graph $G$ the number of 0-bend edges is $k_{0}$ and the number of 2-bend planar edges is $k_{2}^{\prime}$, then there exists a general position 3-D orthogonal drawing of $G$ with $2 m+k_{0}+k_{2}^{\prime}$ bends.

Corollary 1. There exists an infinite family of 6-regular n-vertex graphs, such that in a 2-bend drawing of any of the graphs, $k_{0}+k_{2}^{\prime} \geq 6 n / 7$.

Proof. By Lemma 5 there exists an infinite family of graphs, each with at least $2 m+6 n / 7$ bends in any general position drawing. If there is a 2-bend drawing of such a graph, then by Lemma 8 there is a general position drawing with $2 m+k_{0}+k_{2}^{\prime}$ bends. Hence $2 m+k_{0}+k_{2}^{\prime} \geq 2 m+6 n / 7$ and $k_{0}+k_{2}^{\prime} \geq 6 n / 7$.

The following two results are obtained using the same argument used in the proof of Corollary 1 applied with Lemma 6 and Lemma 7, respectively.

Corollary 2. There exists an infinite family of 6-regular 2-connected $n$-vertex graphs, such that in a 2-bend drawing of any of the graphs, $k_{0}+k_{2}^{\prime} \geq 4 n / 7$.

Corollary 3. There exists an infinite family of 6-regular 4-connected n-vertex graphs, such that in a 2-bend drawing of any of the graphs, $k_{0}+k_{2}^{\prime} \geq 2 n / 7$.

A natural variation of the general position model allows at most two vertices in any one grid-plane with each vertex being coplanar with at most one other vertex. In this model there is at most $n / 2$ pairs of coplanar vertices and hence at most $n / 2$ planar edge routes. Since $n / 2 \leq 4 n / 7$, it follows from Corollary 2 that there exists graphs which do not have 2-bend drawings in this model.

Theorem 9. There exists an infinite family of 2-connected graphs each of which does not have a 2-bend drawing with at most two vertices in any one grid-plane and with each vertex being coplanar with at most one other vertex. 
Acknowledgements: This research was partially completed while a $\mathrm{PhD}$ student in the School of Computer Science and Software Engineering at Monash University, Melbourne, Australia. The author gratefully acknowledges the suggestions of his supervisor Graham Farr, and of Therese Biedl and Antonios Symvonis. In particular, Lemma 6 and Lemma 8 were developed in conjunction with Therese Biedl and Antonios Symvonis, respectively.

\section{References}

1. T. C. Biedl. New lower bounds for orthogonal drawings. J. Graph Algorithms Appl., 2(7):1-31, 1998. 260]

2. M. Closson, S. Gartshore, J. Johansen, and S. K. Wismath. Fully dynamic 3dimensional orthogonal graph drawing. In J. Kratochvil, editor, Proc. Graph Drawing: 7th International Symp. (GD'99), volume 1731 of Lecture Notes in Comput. Sci., pages 49-58, Springer, 1999. 259, 261

3. G. Di Battista, M. Patrignani, and F. Vargiu. A split\&push approach to 3D orthogonal drawing. In Whitesides 10], pages 87-101. 259] 261

4. P. Eades, C. Stirk, and S. Whitesides. The techniques of Komolgorov and Bardzin for three dimensional orthogonal graph drawings. Inform. Proc. Lett., 60(2):97103, 1996. 259, 260

5. P. Eades, A. Symvonis, and S. Whitesides. Three dimensional orthogonal graph drawing algorithms. Discrete Applied Math., 103:55-87, 2000. 259 260, 261261 261, 261,261

6. A. N. Kolmogorov and Ya. M. Barzdin. On the realization of nets in 3-dimensional space. Problems in Cybernetics, 8:261-268, March 1967. 259, 260 261

7. A. Papakostas and I. G. Tollis. Algorithms for incremental orthogonal graph drawing in three dimensions. J. Graph Algorithms Appl., 3(4):81-115, 1999. 259 261 261, 261

8. M. Patrignani and F. Vargiu. 3DCube: a tool for three dimensional graph drawing. In G. Di Battista, editor, Proc. Graph Drawing: 5th International Symp. (GD'97), volume 1353 of Lecture Notes in Comput. Sci., pages 284-290, Springer, 1998. [259] 261

9. R. Tamassia, I. G. Tollis, and J. S. Vitter. Lower bounds for planar orthogonal drawings of graphs. Inform. Process. Lett., 39(1):35-40, 1991. 260.

10. S. Whitesides, editor. Proc. Graph Drawing: 6th International Symp. (GD'98), volume 1547 of Lecture Notes in Comput. Sci., Springer, 1998. [271, 271]

11. D. R. Wood. On higher-dimensional orthogonal graph drawing. In J. Harland, editor, Proc. Computing: the Australasian Theory Symp. (CATS'97), volume 19(2) of Austral. Comput. Sci. Comm., pages 3-8, 1997. 260, 260

12. D. R. Wood. An algorithm for three-dimensional orthogonal graph drawing. In Whitesides [10], pages 332-346. 259, 261, 262, 268, 269

13. D. R. Wood. Lower bounds for the number of bends in three-dimensional orthogonal graph drawings. Technical Report CS-AAG-2000-01, Basser Department of Computer Science, The University of Sydney, 2000. 259262

14. D. R. Wood. Three-Dimensional Orthogonal Graph Drawing. PhD thesis, School of Computer Science and Software Engineering, Monash University, Australia, 2000. 259, 260,261, 261,261, 268 